\title{
Proton Radiation Effects Assessment of a Commercial 12-Megapixel CMOS Imager
}

\author{
Randall Milanowski, Raichelle Aniceto, Fred Hardy, Bert Vermeire, Michael Jacox, Slaven Moro, \\ and Kerri Cahoy
}

\begin{abstract}
Commercial off-the-shelf 12-Megapixel CMOS image sensors were irradiated with $105 \mathrm{MeV}$ protons - one part to a fluence of $4 \times 10^{11}$ protons $/ \mathrm{cm}^{2}$, and a second part to $2 \times 10^{11}$ protons $/ \mathrm{cm}^{2}$. Pixel brightness increases with fluence along with annealing effects are reported. No latch-up events or hangs occurred.
\end{abstract}

\section{INTRODUCTION}

$\mathrm{R}$ adiation-tolerant, high-definition, high-frame-rate, CMOS active pixel sensors (APS) are of interest for spacecraft star tracking, space and earth observation, nuclear imaging, and multitude of other applications. CMOS image sensors are known to be susceptible to single event effects (SEEs), including single event transients (SETs), single event latch-ups (SELs), and single event disturbance effects [1],[2]. The dominant parametric degradation effects are an increase in supply current due to ionizing dose and creation of "hot" pixels (i.e. pixels with high dark current values) due to nonionizing interactions in the pixel sensitive volume [3].

The CMOSIS CMV12000 is a commercial, high-sensitivity pipelined global shutter CMOS image sensor with a resolution of $4096 \times 3072$ pixels [4]. The images integrate 64 LVDS channels, each running at $600 \mathrm{Mbps}$, resulting in a 300 fps frame rate at full resolution with 10 bits per pixel data output or 132 fps with 12 bits per pixel. The sensor is further characterized by 13500 e- full well, 125 e-/sec dark current, 13 e- RMS dark noise, and $90 \mathrm{~dB}$ extended dynamic range. The sensor was not designed for a space environment, though CMOSIS has developed radiation-hardened sensors specifically targeting space applications [5]. In this work, we report radiation test results for CMV12000 subjected to 105 $\mathrm{MeV}$ proton radiation.

Manuscript received July $28^{\text {th }}$, 2017. This work was supported by the Facebook Connectivity Lab.

Randall Milanowski is with M\&A, Inc., 2726 Shelter Island Drive \#268, San Diego, CA, 92106 USA (telephone: +1-619-865-2174, email: rmilanowski@radhard.com).

Raichelle Aniceto is with Facebook. 1 Hacker Way, Menlo Park, CA 94025 USA (telephone: +1-801-859-719, e-mail:raniceto@fb.com).

Fred Hardy, Bert Vermeire, and Michael Jacox are with Space Micro Inc., 10239 Flander Ct. San Diego, CA, 92121 USA (telephone: +1-520-2701816, e-mail: bvermeire@spacemicro.com).

Slaven Moro is with Facebook. 1 Hacker Way, Menlo Park, CA, 94025 USA (telephone: +1-619-300-9204, e-mail: smoro@fb.com).

Kerri Cahoy is with MIT, 77 Massachusetts Ave. Cambridge, MA 02139 USA (telephone: +1-650-814-8148, email: kcahoy@mit.edu).

\section{EXPERIMENTAL APRROACH}

The radiation test campaign was conducted with $105 \mathrm{MeV}$ protons in the BL2C beamline of the TRIUMF cyclotron facility at the University of British Columbia in Vancouver, Canada. The device under test (DUT) was the CMOSIS CV12000 image sensor. The test setup utilized a CMV12000 evaluation kit and comprised of an evaluation board and a Windows 7 PC loaded with a CMOSIS imager configuration software tool written in National Instruments LabView. The LabView program was developed by CMOSIS to facilitate control of the CMV12000 evaluation board. We modified the vendor provided software to streamline the process of capturing and storing full frame, 16-bit "png” format images during radiation testing. The test setup block diagram is shown in Fig. 1.

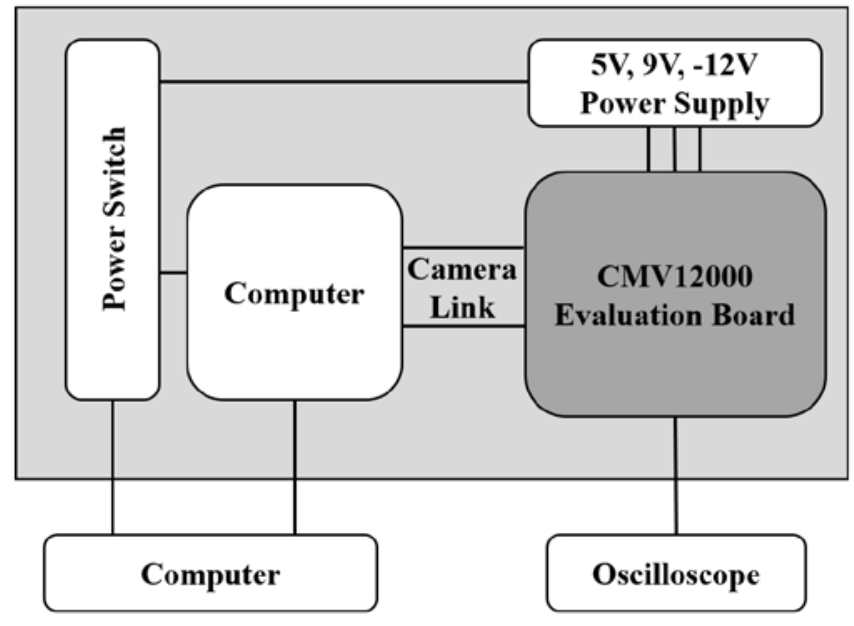

Fig. 1. Test setup block diagram

A GPIB-controlled, triple-output power supply was used to power the evaluation board. The power supply supports slow (1 Hz) monitoring of the supply currents for each of the three outputs. Supply currents were monitored during testing. An oscilloscope was used to monitor a digital test output for indications on non-functionality.

We irradiated two identical image sensor DUTs in different test configurations - "Lens On” and "Cap On”. In both cases the evaluation board was positioned for "backside" exposure with the image sensor was facing away from the beam. 
In the "Lens On" test configuration, DUT 1 was irradiated with a lens on the imager (Fig. 2) and a clock was positioned in the field of view of the imager (Fig. 3). The clock was positioned out of direct line of beam irradiation to minimize proton activation. A block of low density plastic material was used a proton beam stop. Fig. 3 shows a screen image of the CMOSIS configuration software displayed on the computer in the control room, and a recorded image taken by the DUT of the clock in the field of view. Thirty-one separate proton exposure segments were completed on DUT 1 to a total fluence level of $4 \times 10^{11}$ protons $/ \mathrm{cm}^{2}$. The proton irradiation was performed in a series of low fluence segments $\left(\sim 5 \times 10^{8}\right.$ protons $/ \mathrm{cm}^{2}$ ) at a flux of $\sim 2 \times 10^{7}$ protons $/ \mathrm{cm}^{2}$.s until reaching a fluence of $4 \times 10^{10}$ protons $/ \mathrm{cm}^{2}$. The flux was then increased by about a factor of four to $8 \times 10^{7}$ protons $/ \mathrm{cm}^{2} \cdot \mathrm{s}$ in a series of higher fluence exposures $\left(\sim 4.5 \times 10^{10}\right.$ protons $\left./ \mathrm{cm}^{2}\right)$.

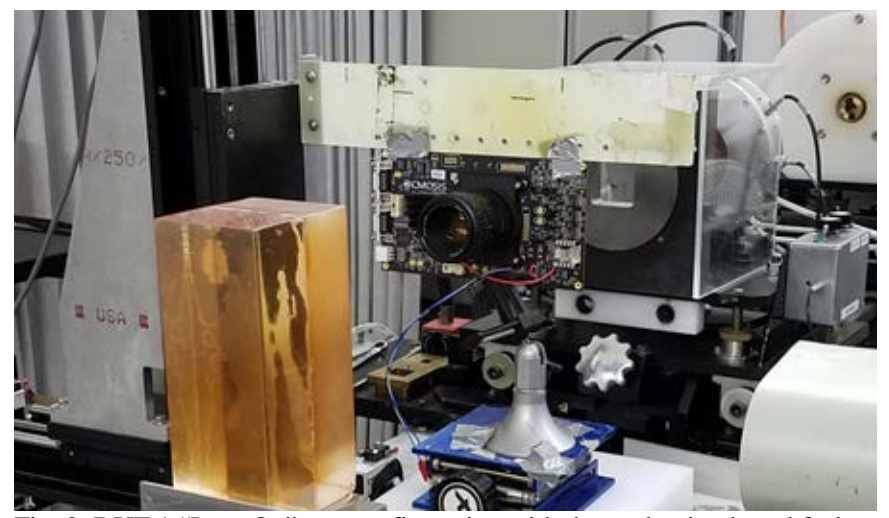

Fig. 2. DUT 1 "Lens On" test configuration with the evaluation board facing away from the proton beam port.

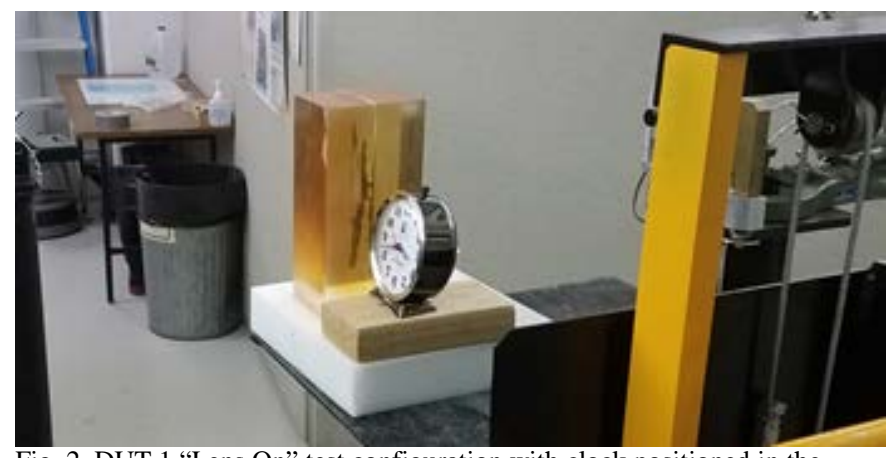

Fig. 2. DUT 1 "Lens On” test configuration with clock positioned in the imager field of view but out of direct line of proton beam irradiation.

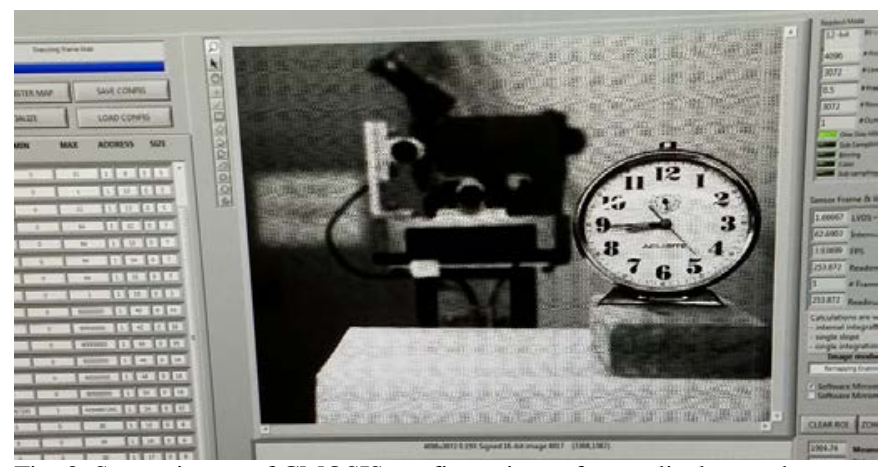

Fig. 3. Screen image of CMOSIS configuration software display on the computer in the control room.
In the "Cap On" test configuration, DUT 2 was irradiated with an opaque cover on the imager and without a lens (Fig. 5 - note: cover is not shown in Fig.). The opaque cover facilitated the characterization of dark current (hot pixels) as a function of fluence. Nine separate proton exposure segments were completed on DUT 2 with flux levels ranging from $\sim 1.5 \times 10^{7}$ protons $/ \mathrm{cm}^{2}$. s to $\sim 7.8 \times 10^{7} \mathrm{protons} / \mathrm{cm}^{2} \cdot \mathrm{s}$ and to a total fluence level of $2 \times 10^{11}$ protons $/ \mathrm{cm}^{2}$. For the preradiation and first seven proton exposures, the DUT was positioned perpendicular to the beam. The last two exposures were performed with the DUT positioned at a 45 degree angle.

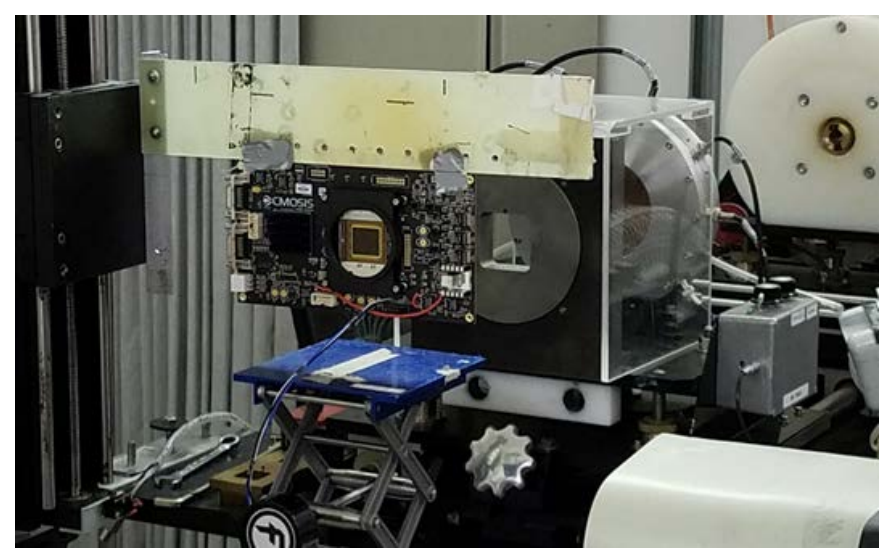

Fig. 5. DUT 2 “Cap On” test configuration. No lens on imager, but an opaque cover was placed on imager for dark current (hot pixel)

characterization. Note that the opaque cover is not shown in this Figure. DUT 2 is oriented for backside exposure for consistency with the DUT 1 "Lens On" configuration.

The DUT functionality was monitored in real-time from the control room of the facility. Still images of the clock were stored while the beam was on (in-situ) and immediately after the beam was turned off (post-run). Image files were also stored prior to irradiation and after an annealing period of 106 days. For each exposure, the proton beam flux, fluence, and exposure time were recorded. The timestamp of each image was also recorded. Fig. 6 shows the timing characteristics of the exposure segments. The diagram indicates the key timing parameters: $\Delta t_{\text {post-run }}, t_{\text {beam_ON }}$, and $t_{\text {beam_OFF }}$.

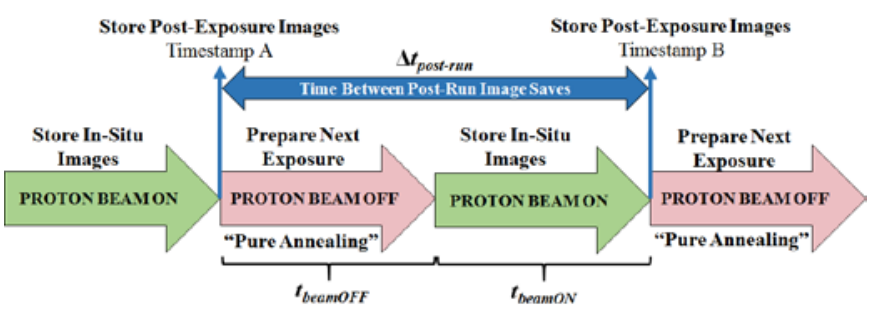

Fig. 6. Diagram for saving in-situ and post-run images during radiation testing. Diagram indicates time between saving post-run images from successive exposures ( $\Delta t_{\text {post-run }}$ ), time duration of proton beam powered on ( $\left.t_{\text {beam_ON }}\right)$, and time duration that proton beam powered off ( $\left.t_{\text {beam_OFF }}\right)$.

Time parameter $\Delta t_{\text {post-run }}$ represents the difference between timestamps of post-run images from consecutive proton exposures. From Fig. 6, $\Delta t_{\text {post-run }}$ would equal the time difference between timestamp A and timestamp B of post-run images. Time parameter $t_{\text {beam_on }}$ represents the time duration 
that the proton beam was powered on and the DUT was irradiated. Some annealing could occur during irradiation of the DUT. Time parameter $t_{\text {beam_ofF }}$ represents the time duration that the proton beam was powered off and the DUT experienced "pure" annealing without irradiation. $t_{\text {beam_OFF }}$ was calculated by subtracting $t_{\text {beam_ON }}$ from $\Delta t_{\text {post-run }}$.

\section{EXPERIMENTAL RESULTS}

\section{A. Lens On Test Configuration}

For the Lens On test configuration, the camera operation was stable during the $105 \mathrm{MeV}$ proton exposures (i.e. realtime display of the clock provided an indicator of correct imager functionality throughout all beam runs).

Pre-radiation images, in-situ images, and post-radiation images for various flux and fluence levels were visually analyzed for differences. In contrast to the pre-radiation image (Fig. 7), the post-exposure image for fluence level of $2 \times 10^{11}$ protons $/ \mathrm{cm}^{2}$ (Fig. 8) contained hot pixels, as seen from the numeral 8 magnified in each image. Lens darkening was also evident between the pre-radiation and post-radiation images.
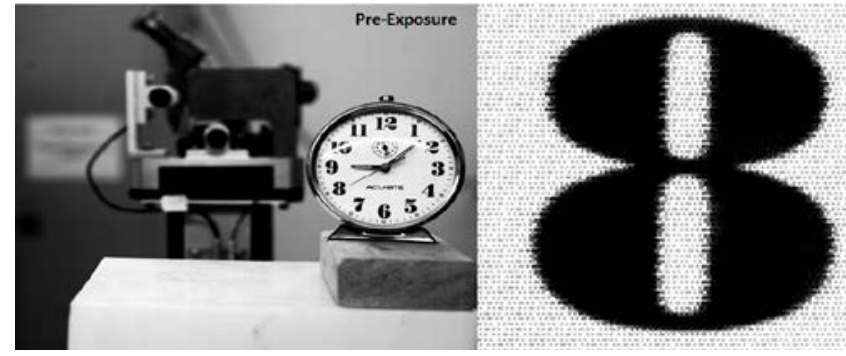

Fig. 7. Pre-exposure image with numeral 8 magnified

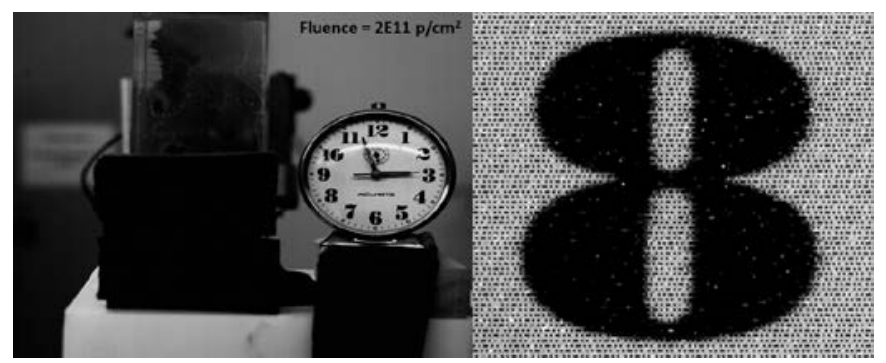

Fig. 8. Image after exposure to fluence of $2 \times 10^{11}$ protons $/ \mathrm{cm}^{2}$. Hot pixels can be seen in the enlarged view of the numeral "8". Lens darkening is also evident in the comparison between pre- and post-exposure images.

Fig. 9 shows the bottom region of in-situ images from flux levels of $1.4 \times 10^{6}$ protons $/ \mathrm{cm}^{2} \cdot \mathrm{s}, 2.20 \times 10^{7} \mathrm{protons} / \mathrm{cm}^{2} \cdot \mathrm{s}$, and $8.3 \times 10^{7}$ protons $/ \mathrm{cm}^{2} \cdot \mathrm{s}$. As proton beam flux increases, there is an increase in photogenerated noise in the images. For the in-situ image with the highest flux level of $8.3 \times 10^{7}$ protons $/ \mathrm{cm}^{2} \cdot \mathrm{s}$ (Fig. 9C), a pattern of circles appears in the image which could be due to shadowing off the package ball grid array. Fig. 10 shows a zoomed in version of in-situ image with flux level of $2.20 \times 10^{7}$ protons $/ \mathrm{cm}^{2}$.s (Fig. 9B) and indicates a high angle particle track crossing $\sim 140$ pixels.

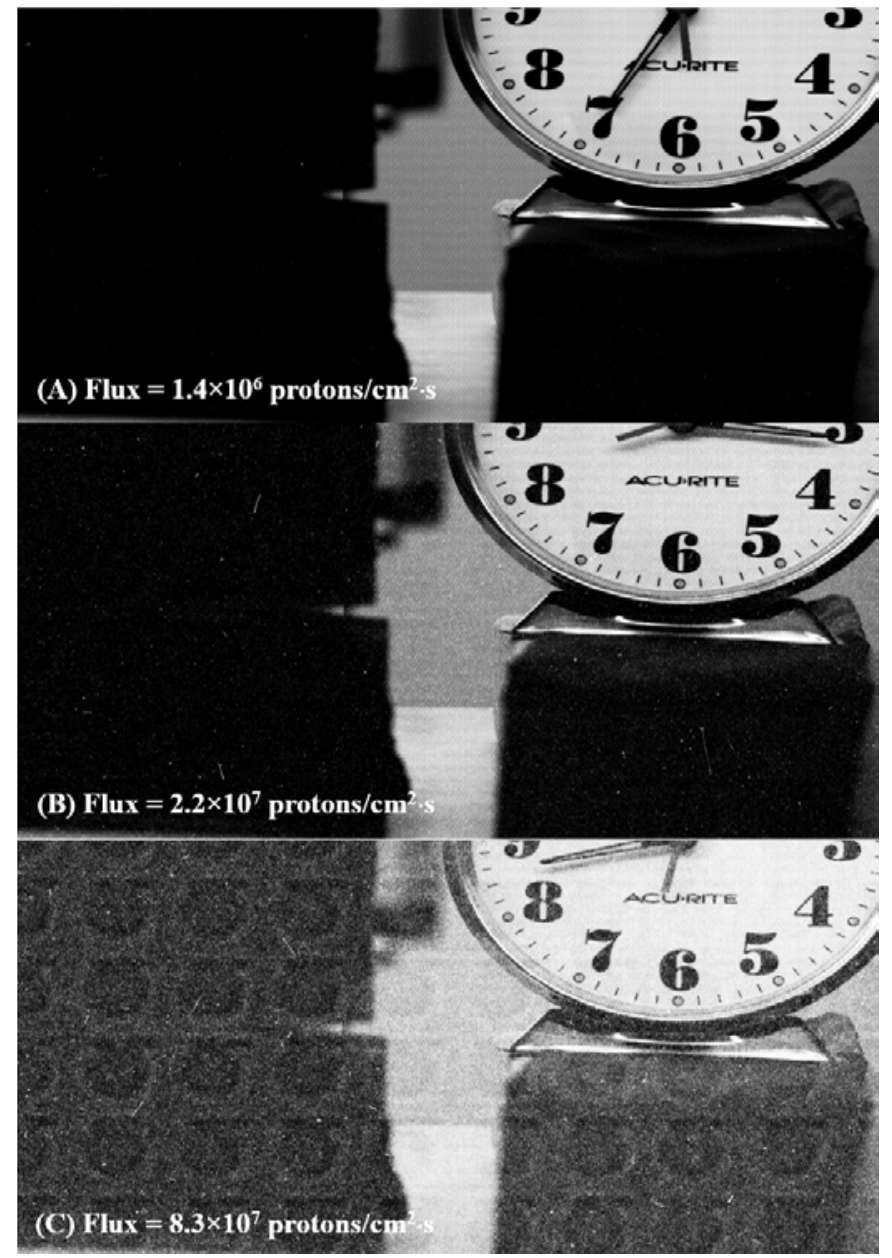

Fig. 9. Images stored while the proton beam is on for flux levels of (A) $1.4 \times$ $10^{6}$ protons $/ \mathrm{cm}^{2} \cdot \mathrm{s}$ (B) $2.20 \times 10^{7}$ protons $/ \mathrm{cm}^{2} \cdot \mathrm{s}$ and (C) $8.3 \times 10^{7}$ protons $/ \mathrm{cm}^{2} \cdot \mathrm{s}$
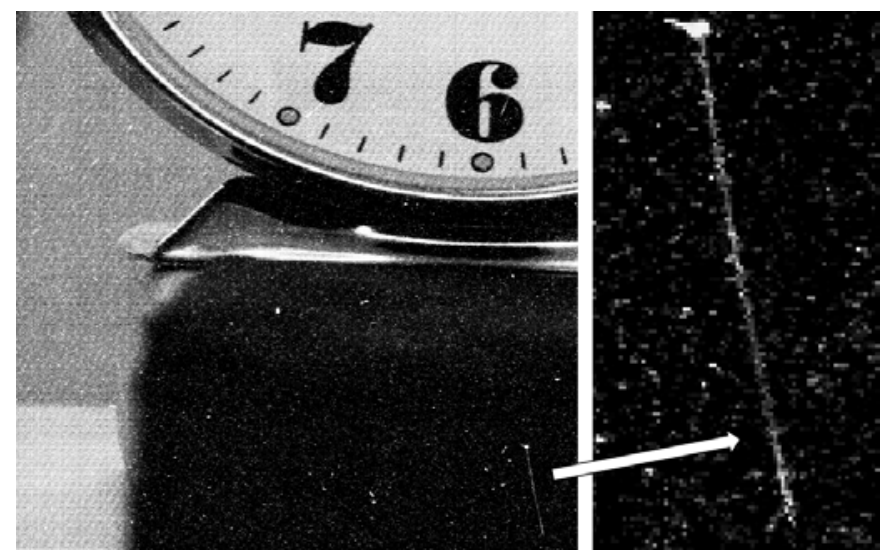

Fig. 10. Zoomed in view of Fig. 9(B) showing a high angle particle track crossing approximately 140 pixels.

The supply current data exhibited no signs of latch-up or other supply current anomalies. This provides some indication of low-LET latch up immunity, however heavy ion testing should also be performed [6]. 


\section{B. Cap On Test Configuration}

A Matlab script analyzed each image file, generated a histogram of the hot pixel count from each image file, and calculated the total number of hot pixels from each image file.

Four pre-radiation images were stored. Three of the preradiation images had $\sim 8$ hot pixels. Fig. 11 shows the histogram for the pixel brightness of a pre-radiation image with 8 hot pixels. The right edge of the histogram has a bar above the white or brightest region of the colormap on the $\mathrm{x}$ axis with 8 hot pixels, and the left edge has a bar above the black or darkest region of the colormap with $\sim 1.2 \times 10^{7}$ pixels.

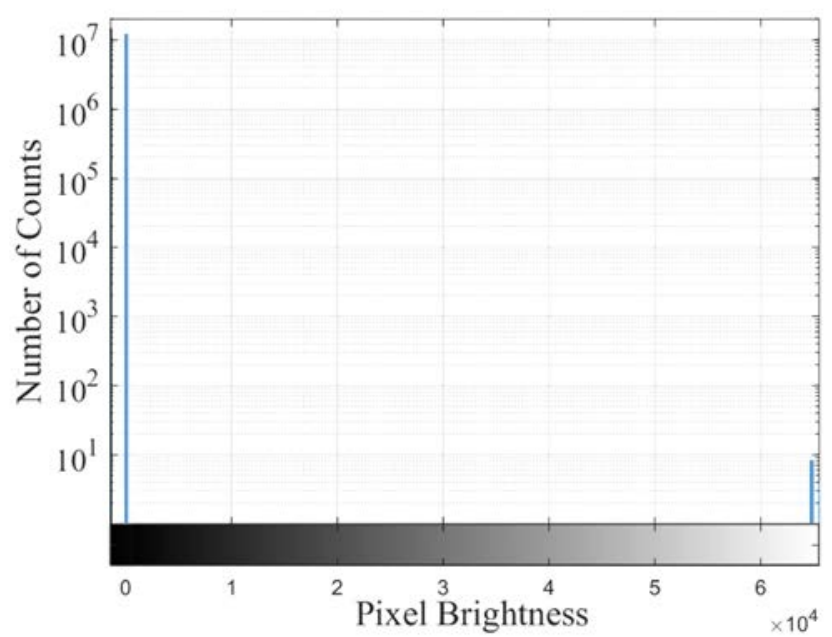

Fig. 11. Pixel brightness histogram for pre-radiation image with 8 hot pixels

One of the four pre-radiation dark field images contained a large number (82954) of "non-zero" pixels. The histogram of the anomalous pre-radiation image is shown in Fig. 12 which reveals spikes in pixel count for a few distinct near-zero pixel brightness levels. This may be a form of fixed pattern noise that occasionally appears in saved images - some post exposure images contained this artifact and required a subtraction process to eliminate the effect from the radiationinduced hot pixel count.

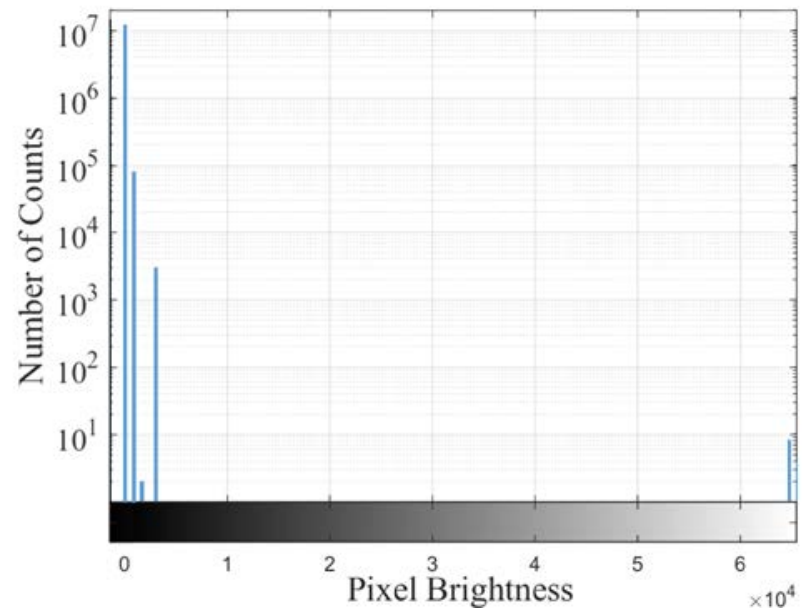

Fig. 12. Pixel brightness histogram for anomalous pre-radiation image
The anomalous pre-radiation image was modified by converting all the black (brightness=0) pixels to white, resulting in a pattern of vertical stripes. The top portion of Fig. 13 shows the resulting image. For a few of the vertical stripes (indicated in Fig. 13, labeled 1-7) the image was magnified to reveal a systematic pattern in the FPN phenomena (lower portion of Fig. 13). Here we note that columns 4 and 5 appear to be mirrored and slightly shifted copies of columns 2 and 3, respectively (2' and 3' denote the mirrored and shifted segments). We also observe that the pattern appears to start over at column 6.

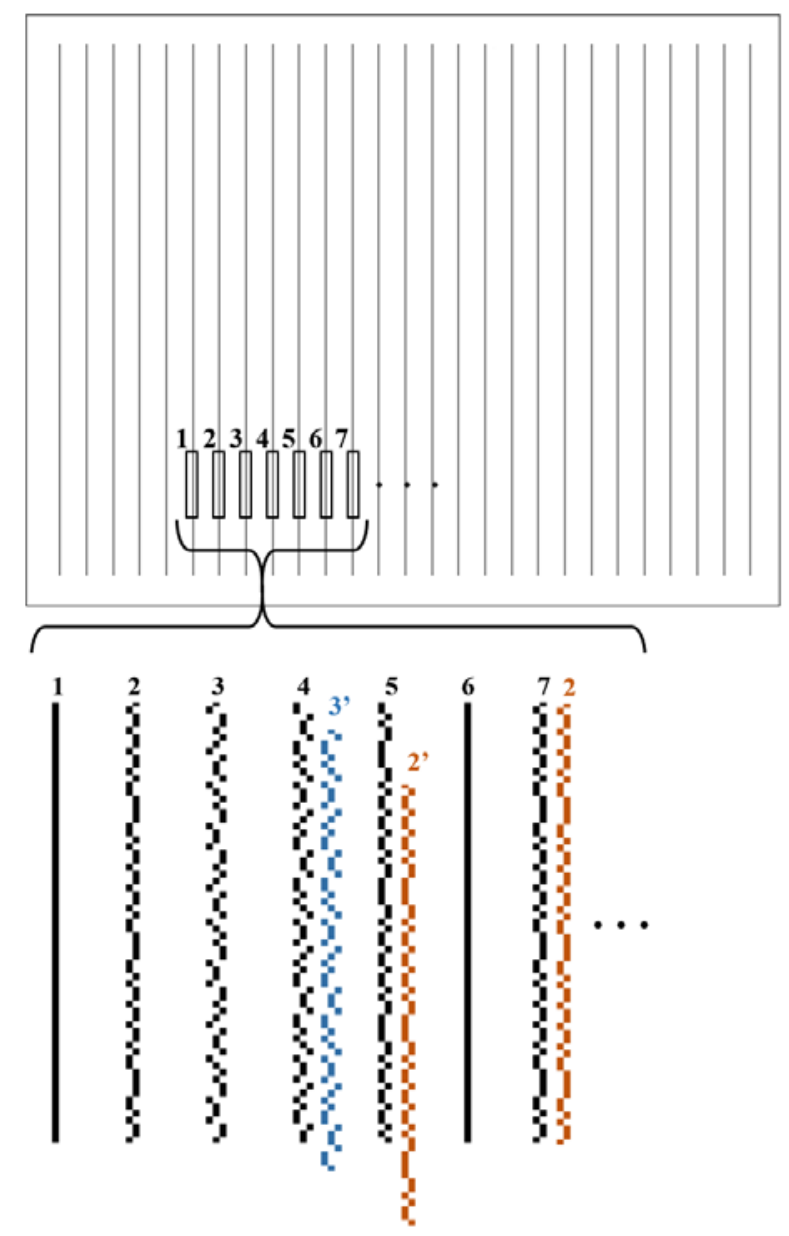

Fig. 13. Analysis of fixed pattern noise in anomalous pre-radiation image. (Top portion) Inverted version of anomalous pre-radiation image with fixed pattern noise of hot pixel vertical stripes. (Bottom portion) A few of the hot pixel vertical stripes are zoomed in and a repetitive pattern is observed among hot pixel columns.

Histograms were also generated for all of the post-exposure image files, and each histogram was visually analyzed for "peaks" similar to the bars from the anomalous pre-radiation image histogram. There were four post-radiation image histograms with the FPN as observed from the anomalous pre-radiation image. Compared to other post-radiation image files in the same set, the "FPN affected" image files had a much higher hot pixel count. We determined a FPN correction factor of 82946 hot pixels by subtracting the nominal pre-radiation hot pixel count (8) from the anomalous pre-radiation hot pixel count (82954). We adjusted the FPN 
affected post-radiation images files by subtracting the FPN correction factor from the hot pixel count. Fig. 14(A) shows an example of a post-radiation exposure \#2 image file with the FPN peaks similar to those from the anomalous preradiation image histogram in Fig. 12. The anomalous preradiation image data set with FPN was subtracted from the post-radiation exposure \#2 image data set to generate a new histogram without the FPN peaks (Fig. 14B). Without FPN correction, the hot pixel count for the anomalous postradiation exposure \#2 image was 83956, and with the FPN correction, the hot pixel count was 1010. The other postradiation exposure \#2 image file (without any anomalous FPN peaks) had a hot pixel count of 1074.

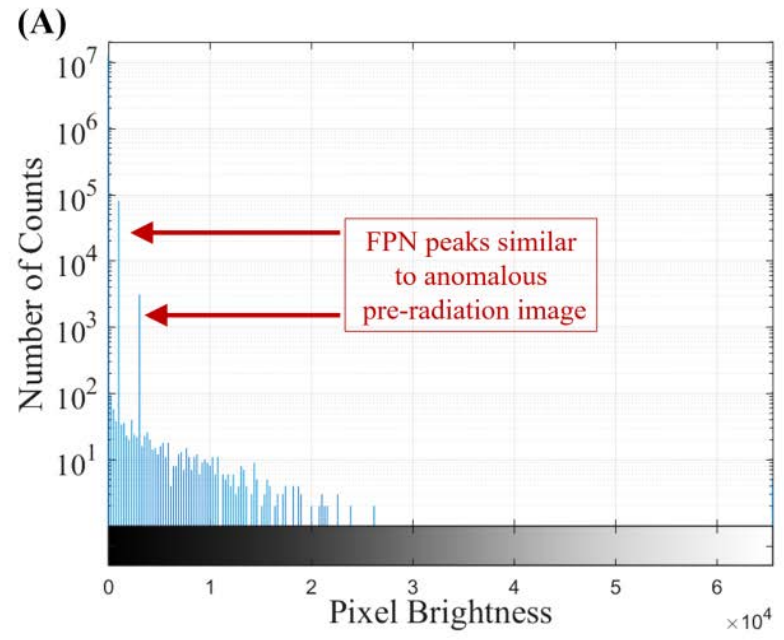

(B)

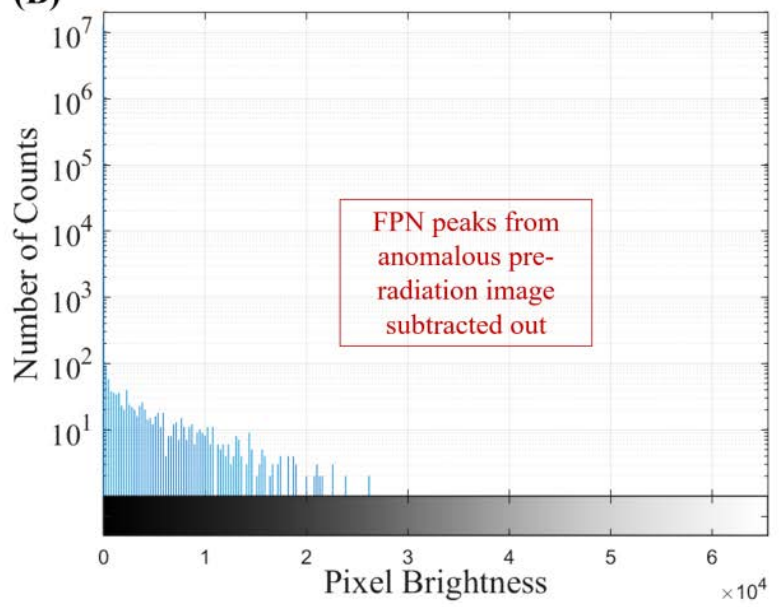

Fig. 14. Example of FPN correction for post-radiation exposure \#2 image file. (A) Histogram with similar FPN peaks as anomalous pre-radiation image. (B) New histogram without FPN peaks, generated by subtracted anomalous pre-radiation image data set from the post-radiation exposure \#2 image data set

A 106 day anneal image file (post total fluence level of $2.0 \times 10^{11}$ protons $/ \mathrm{cm}^{2}$ ) was also observed to have FPN. Fig. 15 shows the inverted version of the FPN affected anneal image with radiation induced hot pixels. Fig. 16 shows the histograms for a post-radiation image file from each of the 9 radiation exposure levels.

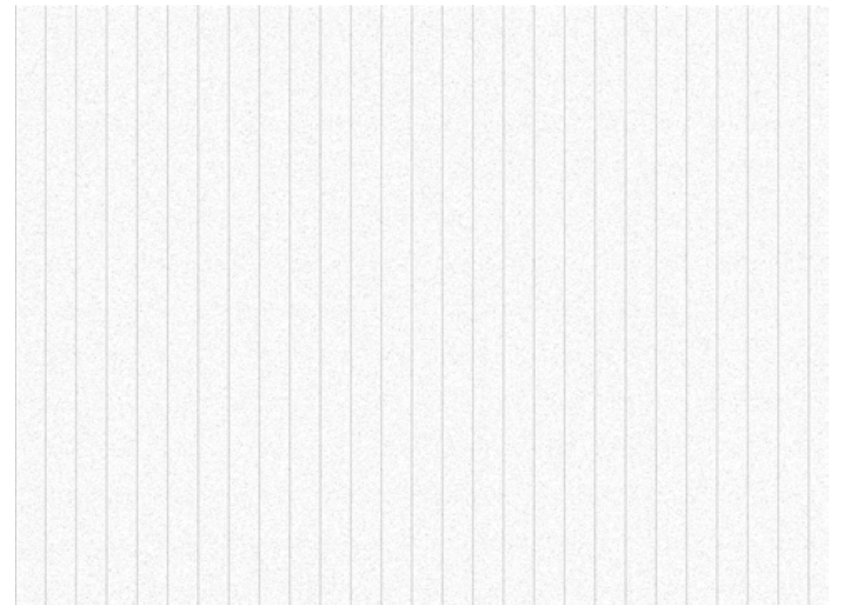

Fig. 15. Inverted image taken after proton fluence $=2 \times 10^{11}$ protons $/ \mathrm{cm}^{2}$ and 106 day anneal unbiased, room temperature anneal. Image shows FPN along with a reduced number of hot pixels.

Significant annealing over the 106-day anneal period was observed through a decrease in the pixel count and a decrease in the distribution for the brightest pixels, compared with the post-radiation exposure \#9 histogram in Fig. 16(I). Fig. 17 shows the pixel histogram for an image file after the 106-day anneal period. 
(A) Post Radiation 1: $1.0 \times 10^{8} \mathrm{p} / \mathrm{cm}^{2}$

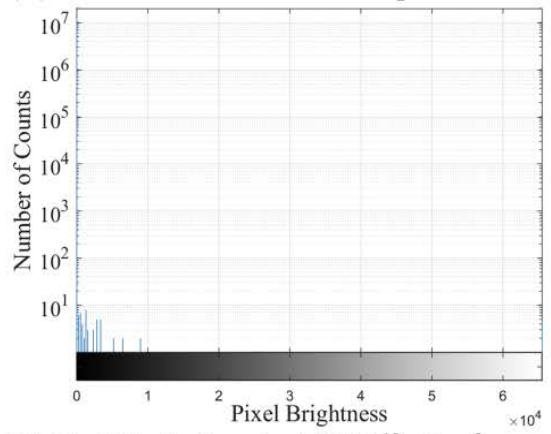

(D) Post Radiation 4: $1.0 \times 10^{10} \mathrm{p} / \mathrm{cm}^{2}$

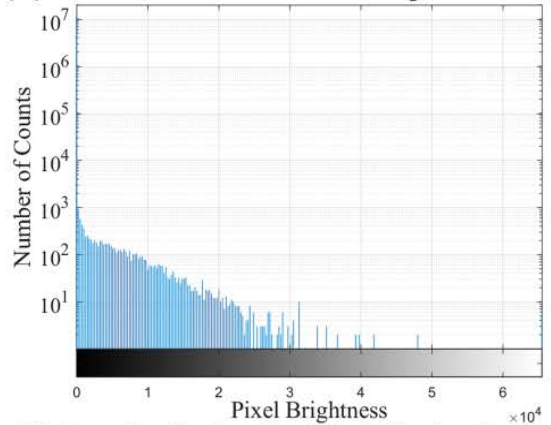

(G) Post Radiation 7: $1.1 \times 10^{11} \mathrm{p} / \mathrm{cm}^{2}$

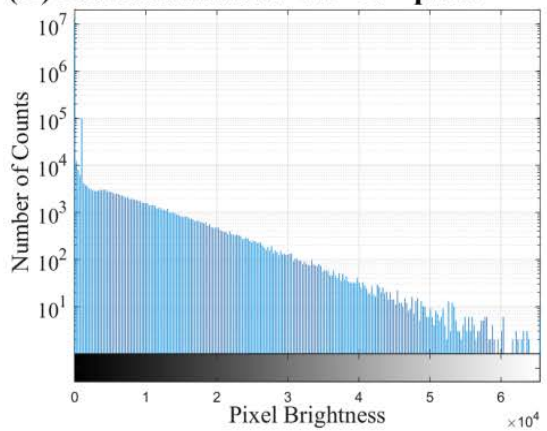

(B) Post Radiation 2: $1.0 \times 10^{9} \mathrm{p} / \mathrm{cm}^{2}$

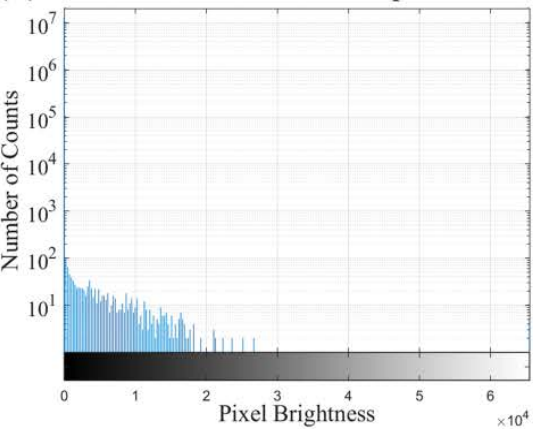

(E) Post Radiation 5: $1.8 \times 10^{10} \mathrm{p} / \mathrm{cm}^{2}$

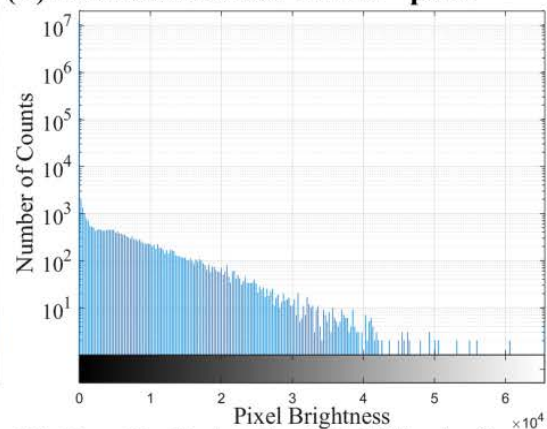

(H) Post Radiation 8: $1.6 \times 10^{11} \mathrm{p} / \mathrm{cm}^{2}$

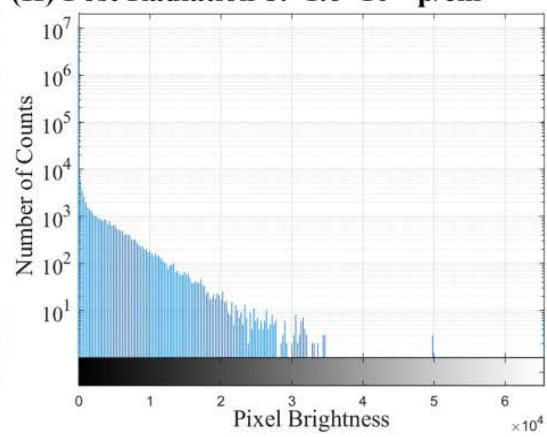

(C) Post Radiation 3: $2.1 \times 10^{9} \mathrm{p} / \mathrm{cm}^{2}$

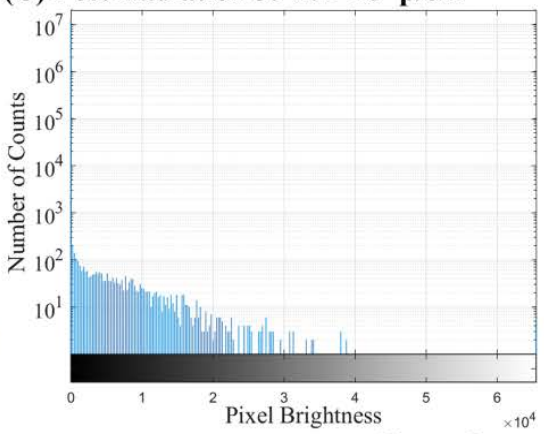

(F) Post Radiation 6: $6.5 \times 10^{10} \mathrm{p} / \mathrm{cm}^{2}$

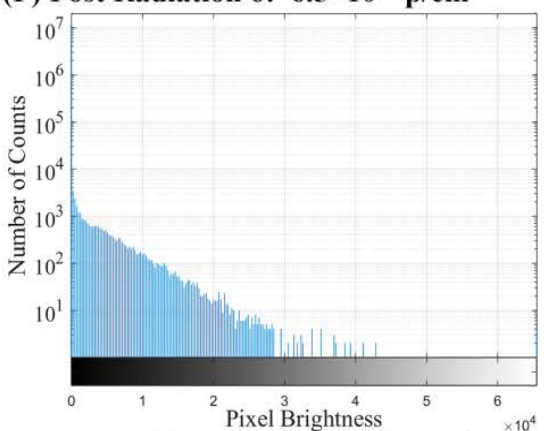

(I) Post Radiation 9: $2.0 \times 10^{11} \mathrm{p} / \mathrm{cm}^{2}$

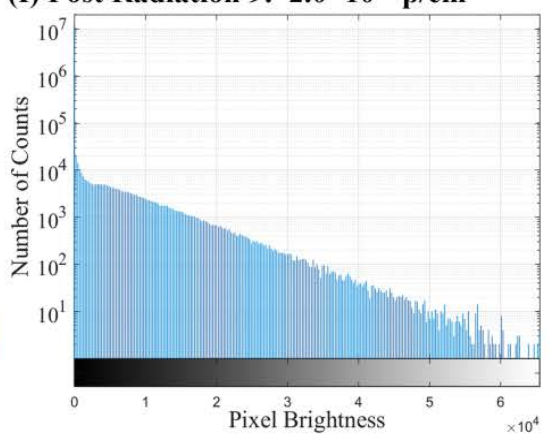

Fig. 16. Pixel histograms for post-radiation image files from each of the 9 proton exposure levels. Exposure level number corresponding proton total fluence are labeled for each histogram.

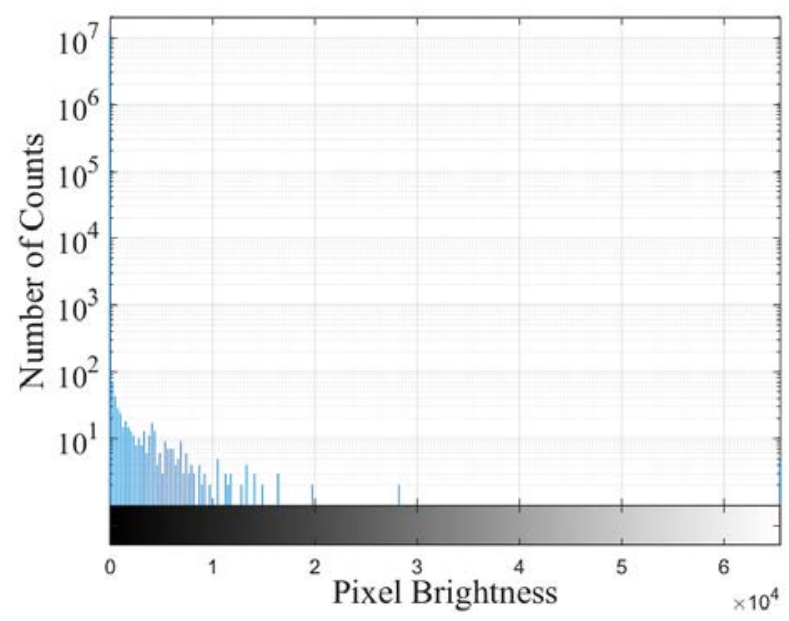

Fig. 17. Pixel histogram for post-anneal period (106 days) image file.

The pre-radiation, post-radiation, and anneal image data are summarized in Table I. For each proton exposure level, the proton beam flux, total fluence level, beam time powered on, and beam time powered off are listed. The average number of hot pixels was calculated for each data set, using the FPN correction factor where necessary. Fig. 18 shows the relationship between total proton fluence and average hot pixel count for the Cap On test configuration data. Beam time on and beam time off are indicated to consider effects of annealing with the results.

TABLE I.

CAP ON TEST CONFIGURATION PRE-RADIATION, POST-RADIATION, AND ANNEAL IMAGE DATA SUMMARY

\begin{tabular}{lccccc}
\hline Data Set & $\begin{array}{c}\text { Flux } \\
{\left[\mathrm{p} / \mathrm{cm}^{2} \cdot \mathrm{s}\right]}\end{array}$ & $\begin{array}{c}\text { Tot. Fluence } \\
{\left[\mathrm{p} / \mathrm{cm}^{2}\right]}\end{array}$ & Avg. Hot Pixels & $\begin{array}{c}\text { tbeam_ON } \\
{[\mathrm{s}]}\end{array}$ & $\begin{array}{c}\text { tbeam_OFF } \\
{[\mathrm{s}]}\end{array}$ \\
\hline Pre-Rad & - & - & $1.0 \times 10^{1}$ & - & - \\
Post-Rad 1 & $1.5 \times 10^{7}$ & $1.0 \times 10^{8}$ & $7.0 \times 10^{1}$ & 7 & 0 \\
Post-Rad 2 & $1.5 \times 10^{7}$ & $1.0 \times 10^{9}$ & $1.0 \times 10^{3}$ & 61 & 177 \\
Post-Rad 3 & $1.6 \times 10^{7}$ & $2.1 \times 10^{9}$ & $2.7 \times 10^{3}$ & 66 & 127 \\
Post-Rad 4 & $7.3 \times 10^{7}$ & $1.0 \times 10^{10}$ & $9.2 \times 10^{3}$ & 112 & 249 \\
Post-Rad 5 & $7.3 \times 10^{7}$ & $1.8 \times 10^{10}$ & $2.6 \times 10^{4}$ & 112 & 119 \\
Post-Rad 6 & $7.4 \times 10^{7}$ & $6.5 \times 10^{10}$ & $2.9 \times 10^{4}$ & 629 & 532 \\
Post-Rad 7 & $7.3 \times 10^{7}$ & $1.1 \times 10^{11}$ & $2.7 \times 10^{5}$ & 630 & 166 \\
Post-Rad 8 & $7.8 \times 10^{7}$ & $1.6 \times 10^{11}$ & $4.2 \times 10^{4}$ & 596 & 1204 \\
Post-Rad 9 & $7.8 \times 10^{7}$ & $2.0 \times 10^{11}$ & $2.8 \times 10^{5}$ & 594 & 71 \\
Anneal & - & - & $5.0 \times 10^{2}$ & - & $9.16 \times 10^{6}$ \\
\hline
\end{tabular}




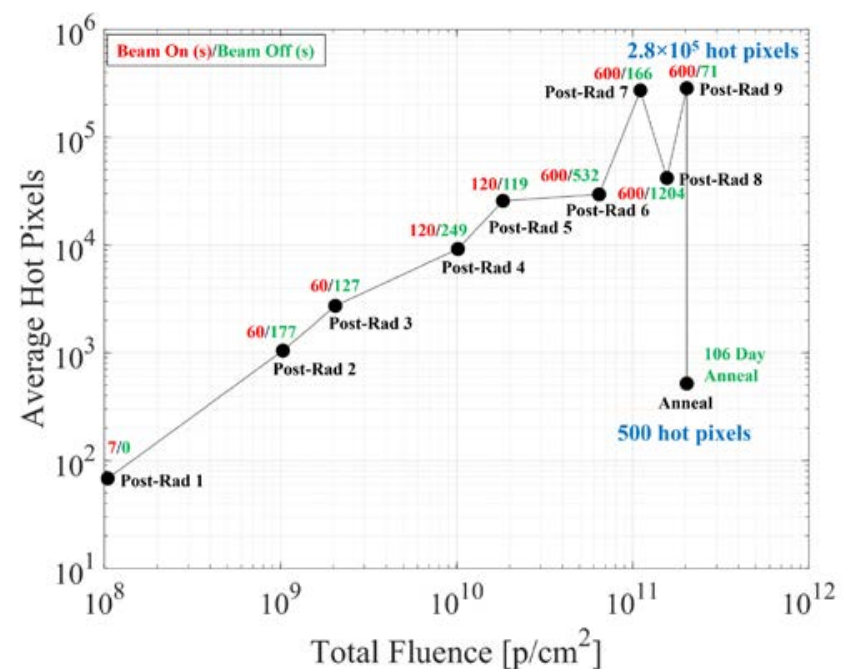

Fig. 18. Total proton fluence versus average hot pixels for each data set.

Beam time powered on and beam time powered off labeled for each data set.

In general, as total proton fluence increased, the average hot pixel count for each data set increased. There was a decrease in the average hot pixel count for post-radiation data set 8 . It should be noted that between radiation exposures \#7 and \#8, there was greater "beam off" time due to adjustment of the DUT to the 45-degree tilt position. Thus, a greater "beam off" time could correlate to hot pixel annealing and explain the decrease in average hot pixel count. Significant annealing over the 106 day anneal period was also observed through a decrease in the average hot pixel count by over two orders of magnitude.

\section{CONCLUSIONS}

SEE susceptibility of a commercial 12-Megapixel CMOS sensor, CMOSIS CMV12000, was assessed with $105 \mathrm{MeV}$ proton test campaign at TRIUMF. No SELs or SEFIs were observed during this test. Hot pixel accumulation with fluence, consistent with well understood displacement damage effects, was characterized for fluences up to $2 \times 10^{11}$ protons $/ \mathrm{cm}^{2}$. An annealing period of 106 days yielded a decrease in the average hot pixel count by over two orders of magnitude.

\section{ACKNOWLEDGMENT}

This work was supported by the Facebook Connectivity Lab.

\section{REFERENCES}

[1] G. R. Hopkinson, "Radiation effects in a CMOS active pixel sensor," IEEE Trans. Nucl. Sci., Vol. 47, No. 6 (2000).

[2] V. Lalucaa, V. Goiffon, P. Magnan, G. Rolland, and S. Petit, "SingleEvent Effects in CMOS Image Sensors," IEEE Trans. Nucl. Sci., Vol. 60, No. 4 (2013).

[3] J. Bogaerts, B. Dierickx, and R. Mertens, "Enhanced Dark Current Generation in Proton-Irradiated CMOS Active Pixel Sensors," IEEE Trans. Nucl. Sci., Vol. 49, No. 3 (2002).

[4] CMV12000 Product sheet; http://www.cmosis.com/products/product_ detail/cmv12000.

[5] X. Wang, J. Bogaerts, W. Ogiers, G. Beeckman, and G. Meynants, "Design and characterization of radiation tolerant CMOS image sensors for space applications,” Proc. Of SPIE, Vol. 8194 (2011).
[6] D. Hiemstra, E. Blackmore, "LET Spectra of Proton Energy Levels from 50 to $500 \mathrm{MeV}$ and Their Effectiveness for Single Event Characterization of Microelectronics," IEEE Trans. Nucl. Sci., Vol. 50, No. 6 (2003). 